ARTICLE

DOI: $10.1038 / s 41467-017-01635-9$

\title{
Graphene-edge dielectrophoretic tweezers for trapping of biomolecules
}

\author{
Avijit Barik ${ }^{1,2}$, Yao Zhang ${ }^{1,3}$, Roberto Grassi ${ }^{1}$, Binoy Paulose Nadappuram ${ }^{4}$, Joshua B. Edel (iD ${ }^{4}$, Tony Low ${ }^{1}$, \\ Steven J. Koester ${ }^{1} \&$ Sang-Hyun $\mathrm{Oh}^{1}$
}

The many unique properties of graphene, such as the tunable optical, electrical, and plasmonic response make it ideally suited for applications such as biosensing. As with other surface-based biosensors, however, the performance is limited by the diffusive transport of target molecules to the surface. Here we show that atomically sharp edges of monolayer graphene can generate singular electrical field gradients for trapping biomolecules via dielectrophoresis. Graphene-edge dielectrophoresis pushes the physical limit of gradient-forcebased trapping by creating atomically sharp tweezers. We have fabricated locally backgated devices with an 8-nm-thick $\mathrm{HfO}_{2}$ dielectric layer and chemical-vapor-deposited graphene to generate $10 \times$ higher gradient forces as compared to metal electrodes. We further demonstrate near-100\% position-controlled particle trapping at voltages as low as $0.45 \mathrm{~V}$ with nanodiamonds, nanobeads, and DNA from bulk solution within seconds. This trapping scheme can be seamlessly integrated with sensors utilizing graphene as well as other twodimensional materials.

\footnotetext{
${ }^{1}$ Department of Electrical and Computer Engineering, University of Minnesota, Minneapolis, MN 55455, USA. ${ }^{2}$ Department of Biomedical Engineering, University of Minnesota, Minneapolis, MN 55455, USA. ${ }^{3}$ Department of Chemistry, University of Minnesota, Minneapolis, MN 55455, USA. ${ }^{4}$ Department of Chemistry, Imperial College London, South Kensington, London SW7 2AZ, UK. Correspondence and requests for materials should be addressed to S.J.K. (email: skoester@umn.edu) or to S.-H.O. (email: sang@umn.edu)
} 
G raphene ${ }^{1}$ is an excellent alternative to noble metals for constructing a wide range of sensors due to its electrical tunability ${ }^{2}$, high quantum efficiency for light-matter interactions ${ }^{3}$, quantum capacitance effects ${ }^{4}, 5$, and tightly confined mid-infrared plasmons ${ }^{6-10}$. Unlike noble metals, such as gold or silver, the carrier concentration in graphene can be tuned, hence enabling the possibility of electrically reconfigurable biosensing ${ }^{11}$. Nanopatterned graphene such as ribbons ${ }^{11}$, nanochannels ${ }^{12}$, or nanopores have all been shown to offer benefits for use in sensing applications. However, biomolecules in viscous media is generally governed by diffusive transport, and the placement of target molecules at the region of highest sensitivity is a key prerequisite to biosensing. Currently, graphene-based biosensors mostly employ DNA or protein immobilization across the entire graphene surface or rely on the random adsorption of biomolecules to the most sensitive regions-the edges or pores in graphene $e^{11,13}$. The ability to precisely position and concentrate target molecules onto the edge of patterned graphene nanostructures is highly desirable yet is not extensively studied. Besides biosensing, such capability can also benefit nanophotonic applications for integrating quantum emitters and plasmonic antennas with tunable optoelectronic properties of graphene $e^{14,15}$.

Various on-chip manipulation techniques, such as optical trapping $^{16-18}$, optoelectronic tweezers ${ }^{19,20}$, flow-through nanopores $^{21}, 22$, electrokinetics ${ }^{23}$, dielectrophoresis $(\mathrm{DEP})^{24}$, or photothermal methods ${ }^{25}$ can be used for the aforementioned purposes. However, it is not trivial to integrate such schemes on patterned graphene chips. We show that the atomic-scale thickness of graphene enables ultra-strong DEP forces for trapping nanoscale objects and molecules along patterned edges of graphene. DEP is a widely used method to manipulate biomolecules or polarizable nanoscale objects by using gradient electrical forces obtained from sharp conducting tips, edges, or small gaps between electrodes ${ }^{24}$. The time-averaged DEP force on a particle of radius $R$ and in a medium of permittivity $\varepsilon_{\mathrm{m}}$ is expressed as ${ }^{24}$

$$
\vec{F}_{\mathrm{DEP}}(\omega)=\pi \varepsilon_{\mathrm{m}} R^{3} \cdot \operatorname{Re}\left(\frac{\varepsilon_{\mathrm{p}}^{*}(\omega)-\varepsilon_{\mathrm{m}}^{*}(\omega)}{\varepsilon_{\mathrm{p}}^{*}(\omega)+2 \varepsilon_{\mathrm{m}}^{*}(\omega)}\right) \nabla|\mathbf{E}|^{2}
$$

where $|\boldsymbol{E}|$ is the magnitude of the electric field, $\varepsilon_{\mathrm{p}}^{*}(\omega)$ and $\varepsilon_{\mathrm{m}}^{*}(\omega)$ are the complex permittivities of the particle and the medium, respectively. For the case where electric field gradient varies greatly over particle dimensions, higher order moments (quadrupole, octopole, and so on) become important ${ }^{26}$. However, in this case as our model system is based on nanoscale particles, we assumed a dipole approximation.

The DEP force depends on the particle volume that goes down with the particle size. However, the thermal Brownian motion becomes very important as we reduce the particle size to submicron length scale. To trap a particle, it is required to overcome a

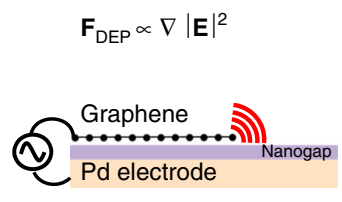

$$
\text { b }
$$
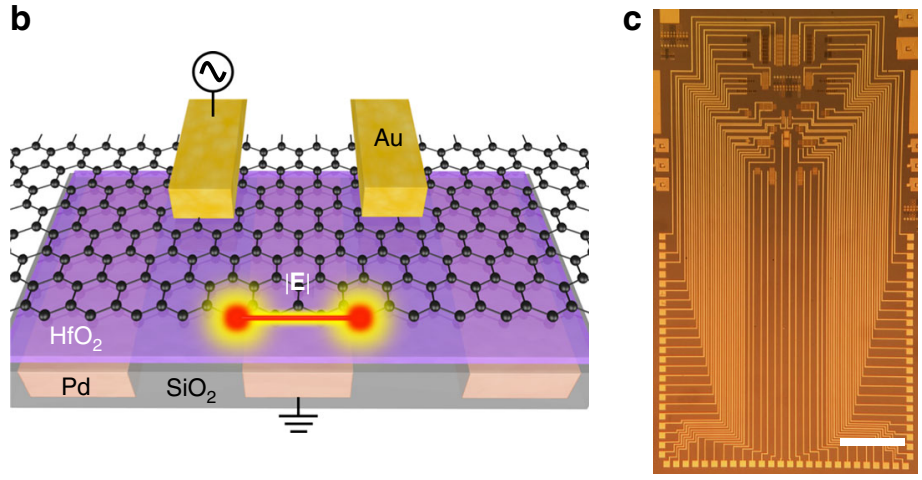

d

Recess etch

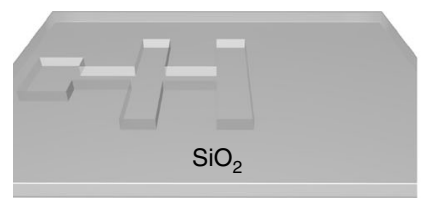

e

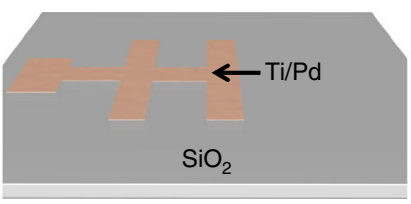

h

Graphene transfer and patterning

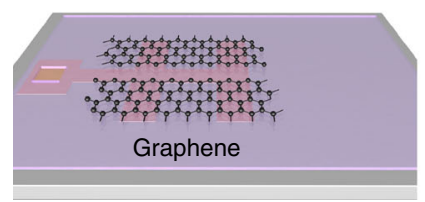

Ohmic contact metallization

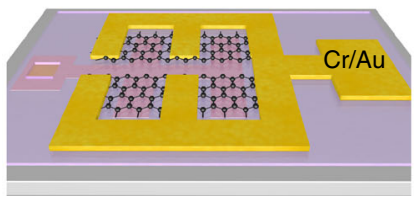

f

$\mathrm{HfO}_{2}$ deposition by ALD

i Contact pad metallization

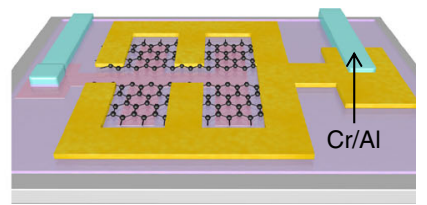

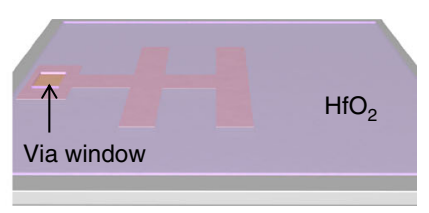

Fig. 1 Graphene as an electrode for dielectrophoresis. a Being a gradient-dependent phenomenon, DEP force is increased as the radius of curvature of an electrode is reduced. The edge of graphene could provide the smallest possible radius of curvature. $\mathbf{b}$ An illustration showing the region of strongest electric field gradient is generated at the intersection of the edge of the graphene by applying an AC bias between the graphene contact electrode (gold) and palladium gate electrode. c A photograph of the chip. Scale bar: $2 \mathrm{~mm}$. d The gate electrode patterns were created on $\mathrm{SiO}_{2}$ substrate by combining photolithography with reactive ion etching and wet etching. e Ti/Pd layer was deposited followed by lift off to create the gate electrodes. $\mathbf{f} 8$-nm-thick $\mathrm{HfO}{ }_{2}$ was deposited to coat the entire surface with an insulator. A via window was created for contact pad metallization with the Pd gate electrode at a later step. g Single-layer graphene grown by chemical vapor deposition process was transferred to the dielectric layer by a wet transfer process, followed by patterning using photolithography and $\mathrm{O}_{2}$ plasma etching. $\mathbf{h}$ Ohmic contacts were made by photolithography, $\mathrm{Cr} / \mathrm{Au}$ deposition by electron-beam evaporation and lift off. i A thick metal layer of $\mathrm{Cr} / \mathrm{Al}$ was added to create low-resistance electrical leads for electrical probing 
a

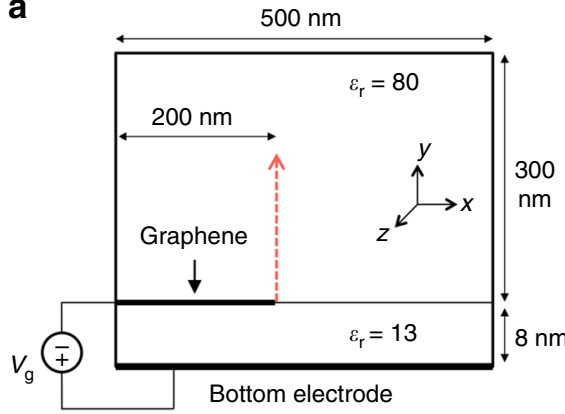

c

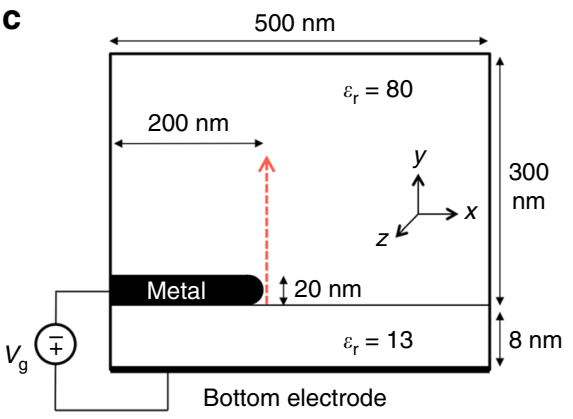

b

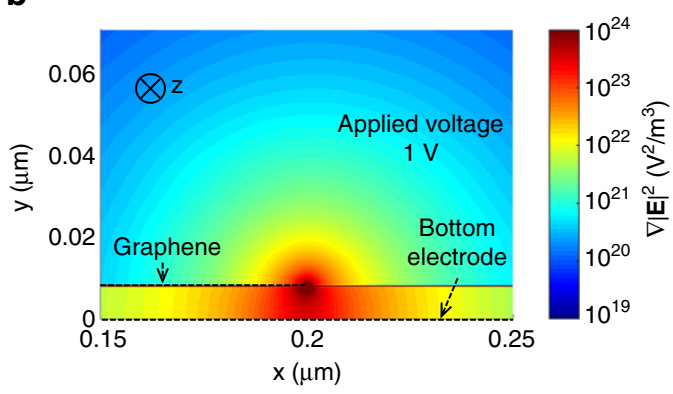

d

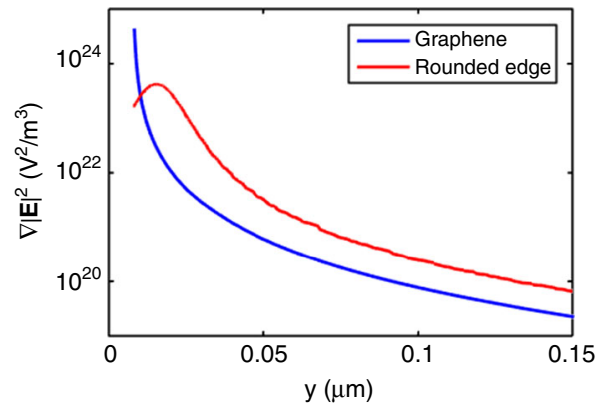

Fig. 2 Self-consistent calculations of field gradients around a graphene edge. a Graphene electrode of zero thickness is placed 8 nm away from the bottom electrode and gradient of electric field intensity is calculated when a bias is applied between the electrodes. $\mathbf{b}$ Intensity plot in logarithmic scale of $\nabla|\mathbf{E}|^{2}$ computed at the back gate voltage $\mathrm{V}_{\mathrm{g}}=1 \mathrm{~V}$ (the flat band voltage is set to zero) showing a strong peak at the graphene edge. An 8 -nm-thick $\mathrm{HfO}_{2}$ layer with a dielectric constant of 13 was assumed between the electrodes. The rest of the simulation domain was assumed to be water with a dielectric constant of 80. c Vertical cut-line (noted by red arrow) of the intensity plot in a at $x=0.2 \mu \mathrm{m}$ (position of the graphene edge), compared with the result of a similar simulation where graphene is replaced by a metal with a thickness of $20 \mathrm{~nm}$ and a realistic round edge with radius of curvature $10 \mathrm{~nm}$. $\mathbf{d}$ The two profiles are similar but the magnitude of $\nabla|\mathbf{E}|^{2}$ at the edge position is greatly enhanced in the graphene case because of its one-atom thickness

the thermal force, $F_{\mathrm{T}}$, given by

$$
F_{\mathrm{T}}=\frac{k_{\mathrm{B}} T_{\mathrm{R}}}{2 R}
$$

where $T_{\mathrm{R}}$ is the room temperature and $k_{\mathrm{B}}$ is the Boltzmann constant.

Since the DEP force is proportional to the field gradient term $\nabla|\mathbf{E}|^{2}$, it is a scalable particle trapping method, i.e., reducing the radius of curvature of electrode features (e.g., tip $\mathrm{s}^{27}$ ) or electrodeto-electrode separation ${ }^{28}$ can significantly boost its magnitude. Patterned metallic nanoelectrode ${ }^{29}$ or bottom-up structures such as isolated carbon nanotubes $(\mathrm{CNTs})^{30,31}$ have been used to shrink the critical dimension of DEP electrodes into single-digit nanometer scale. Extrapolating this idea further toward the ultimate limit, we conceive a design to turn Angstrom-scalethickness monolayer graphene into an electrode that provides the sharpest possible edge over millimeter length scales, which cannot be created using lithographically patterned metal nanostructures or even CNTs. Although single-walled CNT electrodes can provide sharp nanometer-scale edges, these devices often suffer from inability to control the gap between CNT electrode and the gate, as well as difficulty to form high-density regular arrays of CNTs. Previously, Xie et al. ${ }^{32}$ used an atomic force microscopy probe to cut graphene into small-area interdigitated electrodes and showed DEP trapping of CNTs over these structures.

In this work, we demonstrate a novel scheme to turn exposed edges of graphene into strong DEP trapping sites. This was done by constructing a robust large-scale graphene-edge DEP platform and trapping nanoparticles and biomolecules at the edge of this monolayer graphene atop an 8-nm-thick insulator with low bias voltages. Such capability can offer graphene-based sensors a unique solution to diffusion-limited mass transport problems ${ }^{33}$.

\section{Results}

Design and fabrication of a graphene DEP chip. Being a gradient-field dependent phenomenon, DEP is greatly enhanced by reducing the radius of curvature of the electrode, with graphene edge providing the sharpest tip (Fig. 1a). Reducing the gap between the graphene and the ground electrode to sub- $10 \mathrm{~nm}$ length scales aids in further enhancement of the DEP forces. A schematic of the device design is illustrated in Fig. 1b. Lithographically patterned graphene films were created as electrodes for DEP experiments. Applying an alternating current (AC) bias between the graphene contact electrode $(\mathrm{Au})$ and the $\mathrm{Pd}$ gate electrode, generates ultra-high electric field gradients near the graphene edge that can be used to trap nanoparticles by DEP. The strength of this electric field gradient depends on the gap between the two electrodes, which in this case is $8 \mathrm{~nm}$ (thickness of the dielectric layer $\left(\mathrm{HfO}_{2}\right)$ ). A photograph of the chip is shown in Fig. 1c, which was prepared by employing a multi-step fabrication process (Fig. $1 \mathrm{~d}-\mathrm{i})$. First, a metal electrode $(\mathrm{Ti} / \mathrm{Pd})$ pattern was created on a Si wafer with a thick layer of $\mathrm{SiO}_{2}$ on it. Next, the entire wafer surface, including the Pd electrode, was coated with 8-nm-thick $\mathrm{HfO}_{2}$ deposited by atomic layer deposition (ALD). Then, single-layer graphene grown by chemical vapor deposition (CVD) was transferred onto the wafer and etched into rectangular patterns. In addition to the sharpness of the graphene edge, the nanoscale gap between graphene and the Pd gate electrode (defined by the thickness of the $\mathrm{HfO}_{2}$ layer) further enhances the electric field. Finally, photolithography was employed to pattern $\mathrm{Cr} / \mathrm{Au}$ contact electrodes, which formed Ohmic contacts to the graphene. The gate and the contact electrodes were arranged in 

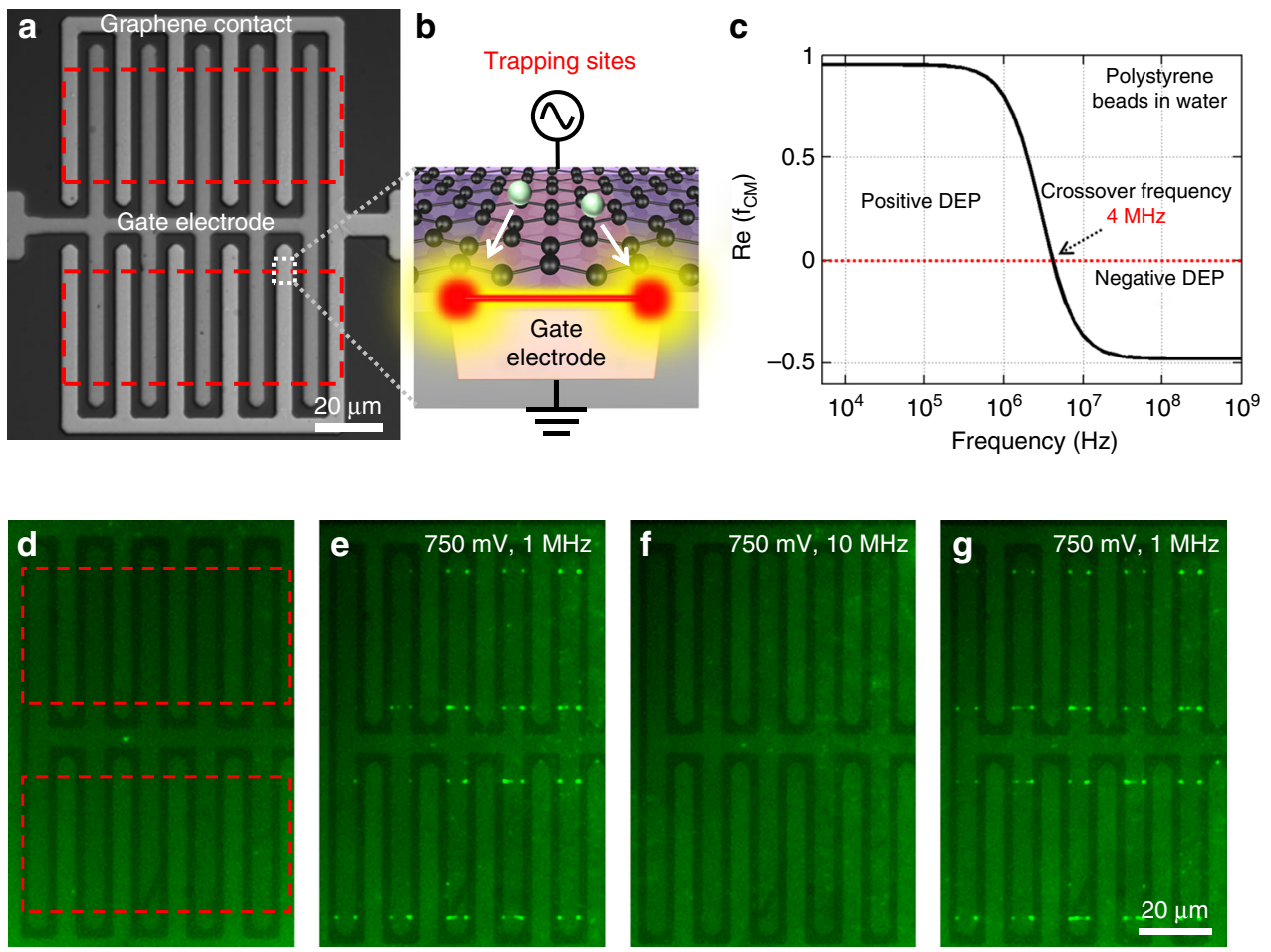

Fig. 3 DEP manipulation of $190 \mathrm{~nm}$ polystyrene beads using graphene electrode. a A bright field microscopic image of the electrode design is shown with the location of the graphene patterns marked with red rectangles. $\mathbf{b}$ Applying an AC bias between graphene contact and the bottom gate electrode creates strong field gradients at the graphene edge. However, the region of strongest field strength resides at the intersection points of the graphene edge and the bottom electrode edge (red circles) - "trapping sites". c Clausius-Mossotti (CM) factor plot for polystyrene beads in water with a conductivity of $4 \mu \mathrm{S} / \mathrm{cm}$. d A green background was observed before applying voltage from the bulk solution. $\mathbf{e}-\mathbf{g} 190 \mathrm{~nm}$ polystyrene beads were trapped $\mathbf{e}$ at the trapping sites due to positive DEP $(750 \mathrm{mV}, 1 \mathrm{MHz})$. The beads can also be released $\mathbf{f}$ due to negative $\mathrm{DEP}(750 \mathrm{mV}, 10 \mathrm{MHz})$ and then retrapped again $\mathbf{g}$ by applying positive DEP

an interdigitated fashion to minimize series resistance and to produce more exposed edges efficiently trap the nanoparticles and molecules. Details of the fabrication process are elaborated in Methods section.

The key feature of our processing scheme is the wafer-scale throughput and scalability of trap arrays. In the layout we employed, the gate finger spacing is $15 \mu \mathrm{m}$ with two graphene segments on either side, and these segments have a pitch of 60 $\mu \mathrm{m}$. The current design provides a density of four trapping sites per $900 \mu \mathrm{m}^{2}$. This density can be further enhanced by reducing the pitch of the gate fingers as well as the graphene segments to 1 $\mu \mathrm{m}$, which is realistic using i-line optical lithography. In such a case, the trapping site density could be increased to 4 sites per 1 $\mu \mathrm{m}^{2}$, a nearly $1000 \times$ improvement over the current devices. Furthermore, using advanced optical lithography or electronbeam lithography to form the gate fingers and segment the graphene, the trapping density could be further increased by another 1-2 orders of magnitude.

Self-consistent calculations of graphene-edge electrostatic fields. We calculated the gradient of the electric field intensity $\left(\nabla|\mathbf{E}|^{2}\right)$, which is responsible for DEP trapping, for a semi-infinite graphene "electrode", electrically biased by a metal gate electrode, as shown in Fig. 2a. An 8-nm-thick $\mathrm{HfO}_{2}$ layer with a dielectric constant of 13 is assumed between the electrodes. As the experiments were all performed in water, the rest of the simulation domain is assumed to be water with a dielectric constant of 80. The bottom electrode is treated as an ideal metal and graphene is modeled as a layer with zero thickness. An illustration of the simulation domain is shown in Fig. 2a. Here we note that graphene is not a perfect metal, due to its relatively small electronic density-of-states. The electrostatics problem then requires a self-consistent solution of Poisson's equation and that of the finite charge density within graphene, as imposed by its Dirac-like energy dispersion:

$$
\begin{gathered}
\nabla \cdot(\varepsilon \nabla \varphi)=e n \delta\left(y-y_{0}\right) \\
n(x)=\frac{2}{\pi}\left(\frac{k_{\mathrm{B}} T_{\mathrm{R}}}{\hbar v_{\mathrm{F}}}\right)^{2}\left[\mathcal{F}_{1}\left(\frac{\mu}{k_{\mathrm{B}} T_{\mathrm{R}}}\right)-\mathcal{F}_{1}\left(\frac{-\mu}{k_{\mathrm{B}} T_{\mathrm{R}}}\right)\right], \quad x<200 \mathrm{~nm}
\end{gathered}
$$

The symbols are defined as follows: $\varphi(x, y)$ is the $2 \mathrm{D}$ electrostatic potential, $n(x)$ the net electron concentration (per unit area) on the graphene layer, $\varepsilon=\varepsilon_{\mathrm{r}} \varepsilon_{0}\left(\varepsilon_{r}\right.$ is the dielectric constant of the different media and $\varepsilon_{0}$ the vacuum permittivity), $\delta$ is Dirac's delta function, $y_{0}=8 \mathrm{~nm}$ the vertical position of the graphene layer, $\hbar$ the reduced Planck constant, $\nu_{\mathrm{F}} \approx 10^{6} \mathrm{~m} / \mathrm{s}$ the graphene Fermi velocity, $\mathcal{F}_{1}$ the Fermi-Dirac integral of order 1 , and $\mu(x)$ the position-dependent chemical potential. The latter, in turn, is given as:

$$
\mu(x)=e \varphi\left(x, y=y_{0}\right), \quad x<200 \mathrm{~nm}
$$

We applied the Dirichlet boundary condition $\varphi=V_{\mathrm{g}}=1 \mathrm{~V}$ at the bottom edge of the simulation domain and Neumann boundary conditions everywhere else. The gradient of $|\mathbf{E}|^{2}$ is computed from $\varphi$ with finite differences method.

We have performed frequency-dependent capacitance measurements on metal-insulator-metal capacitors using our ALD 

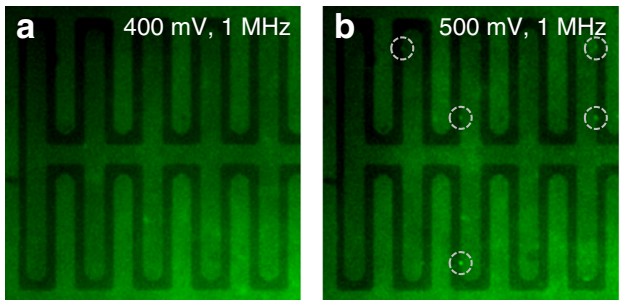

e

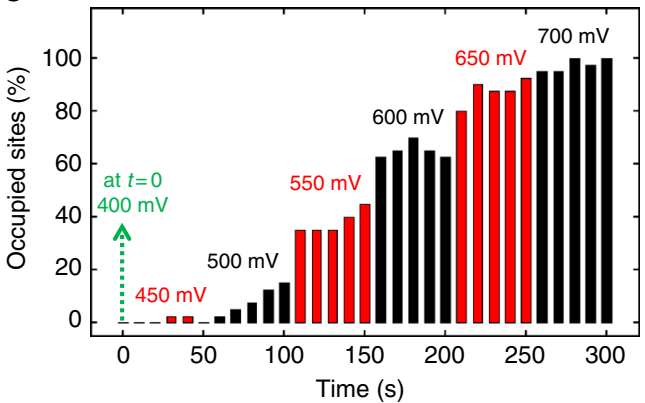

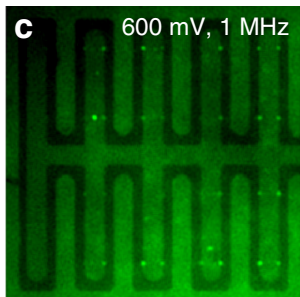

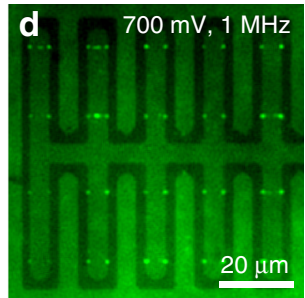

$\mathbf{f}$

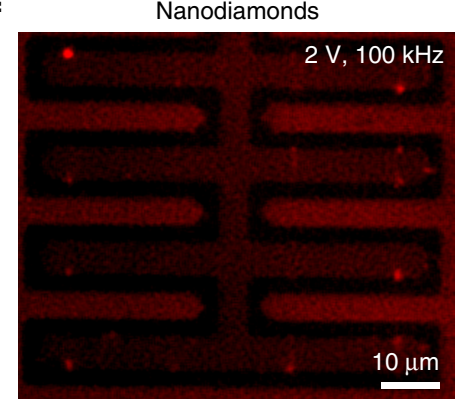

Fig. 4 Voltage dependence and nanodiamond trapping. a-d Applied voltage was gradually increased at a frequency of $1 \mathrm{MHz}$ and a greater number of trapped beads was observed at the trapping sites. e The occupancy (\%) of the trapping sites was monitored as the applied bias amplitude was increased. The $V_{\min }$ for $190 \mathrm{~nm}$ polystyrene beads was $500 \mathrm{mV}$. f Nanodiamond particles were trapped at the trapping sites by applying $2 \mathrm{~V}$ and $100 \mathrm{kHz}$ frequency

$\mathrm{HfO}_{2}$ dielectrics and observed only about $\sim 10 \%$ frequency dispersion between $1 \mathrm{kHz}$ and $10 \mathrm{MHz}$. These results are consistent with previous results ${ }^{34}$ for ALD-deposited $\mathrm{HfO}_{2}$, and further shows that our dielectrics do not display significant dielectric relaxation as is observed in rare-earth-doped and other more complex high-k dielectrics ${ }^{35}$.

The $\nabla|\mathbf{E}|^{2}$ field gradient map is shown in Fig. $2 b$ for a $1 \mathrm{~V} \mathrm{DC}$ bias applied between the gate and graphene electrodes. The $\nabla|\mathbf{E}|^{2}$ value is highest at the edge of the graphene electrode, which acts as a hotspot for DEP trapping. A vertical cut-line of the $\nabla|\mathbf{E}|^{2}$ field gradient at the position of the graphene edge is plotted and compared to a case where the graphene electrode was replaced with a realistic metal electrode of $20 \mathrm{~nm}$ thickness and $10 \mathrm{~nm}$ radius of curvature (Fig. 2c). The maximum value of $\nabla|\mathbf{E}|^{2}$ at the graphene edge is about an order of magnitude higher as compared to the metal electrode (Fig. 2d). However, the effect of the graphene edge becomes less pronounced as we move away from the boundary. In the bulk solution (away from the electrode boundary), the effect of DEP is similar in both cases and mostly dependent on the gap between the electrodes ( $8 \mathrm{~nm}$ here). However, near the graphene edge, gradient forces are stronger than the metal electrode, demonstrating its capability for larger short-range trapping. This in turn enables trapping of small number of analyte nanoparticles in a more controllable fashion at the edge of the graphene electrode without much interference from the bulk solution. For protein molecules or quantum dots that are typically $<10 \mathrm{~nm}$ in diameter, graphene electrodes could provide a stronger trapping force to hold them on to the electrode edge.

Experimental demonstration of graphene-edge DEP trapping and repulsion. A bright-field optical microscope image of the final device is shown in Fig. 3a, where the locations of the graphene films are noted by red rectangles. Applying an AC bias between the gate and graphene contact electrodes creates a region of strong electric field gradient near the graphene edge, with the maximum $\nabla|\mathbf{E}|^{2}$ at either end of the graphene electrode boundary (noted by red circles in Fig. 3b), where graphene edges intersect with buried metal electrodes running in an orthogonal direction. These point junctions act as the region of strongest trapping potential because the $\nabla|\mathbf{E}|^{2}$ is enhanced by non-uniform fields of both the graphene and the gate electrode edges. A particle under the influence of DEP is driven toward these junctions, also termed as the "trapping site". However, the entire boundary of the graphene electrode may also act as a DEP trap, albeit to a lesser extent than the trapping sites. The magnitude of the DEP force depends on the sharpness of the electrode boundaries as well as the $8 \mathrm{~nm}$ gap between the electrodes.

Polystyrene beads, $190 \mathrm{~nm}$ in diameter, were used to demonstrate efficient DEP trapping and releasing with sub-1 V bias voltages. The polarity of the DEP force depends on the frequency-dependent Clausius-Mossotti (CM) factor $\left(f_{\mathrm{CM}}(\omega)\right)$ given by

$$
f_{\mathrm{CM}}(\omega)=\left(\frac{\varepsilon_{\mathrm{p}}^{*}(\omega)-\varepsilon_{\mathrm{m}}^{*}(\omega)}{\varepsilon_{\mathrm{p}}^{*}(\omega)+2 \varepsilon_{\mathrm{m}}^{*}(\omega)}\right)
$$

Particles are attracted toward the trapping sites by positive DEP $\left(\operatorname{Re}\left(f_{\mathrm{CM}}(\omega)\right)>0\right)$ or are repelled away by negative DEP $\left(\operatorname{Re}\left(f_{\mathrm{CM}}(\omega)\right)<0\right)$. The frequency of the applied AC bias at which such transition takes place, $f_{\mathrm{CM}}(\omega)=0$, is called the crossover frequency. To predict the frequency response of the polystyrene beads in water (conductivity $=4 \mu \mathrm{S} / \mathrm{cm}$ ), the $\mathrm{CM}$ factor was plotted as a function of frequency (Fig. 3c). The crossover frequency is found to be $4 \mathrm{MHz}$. Hence, a frequency of $1 \mathrm{MHz}$ was chosen for positive DEP and $10 \mathrm{MHz}$ for negative DEP. Fluorescently labeled polystyrene beads $\left(\lambda_{\mathrm{ex}}: 470 \mathrm{~nm}, \lambda_{\mathrm{em}}: 525\right.$ $\mathrm{nm})$ were used to visualize the DEP manipulation capability of the graphene electrodes using a $\times 50$ objective. Bulk fluorescence was observed before applying a voltage, which comes from the surrounding solution containing fluorescent beads (Fig. 3d). As soon as a bias of amplitude $750 \mathrm{mV}$ (single-sided) was turned on, beads were selectively trapped at the trapping sites, which provide the maximum DEP force as discussed above (Fig. 3e). Of the 40 available trapping sites (4 sites on each finger electrode), 34 (85\%) of them were seen to be occupied. The trapped particles could be released due to negative DEP, simply by switching the frequency to $10 \mathrm{MHz}$ (Fig. 3f). Particles were trapped again by switching back to the positive DEP mode - showing the reversible 

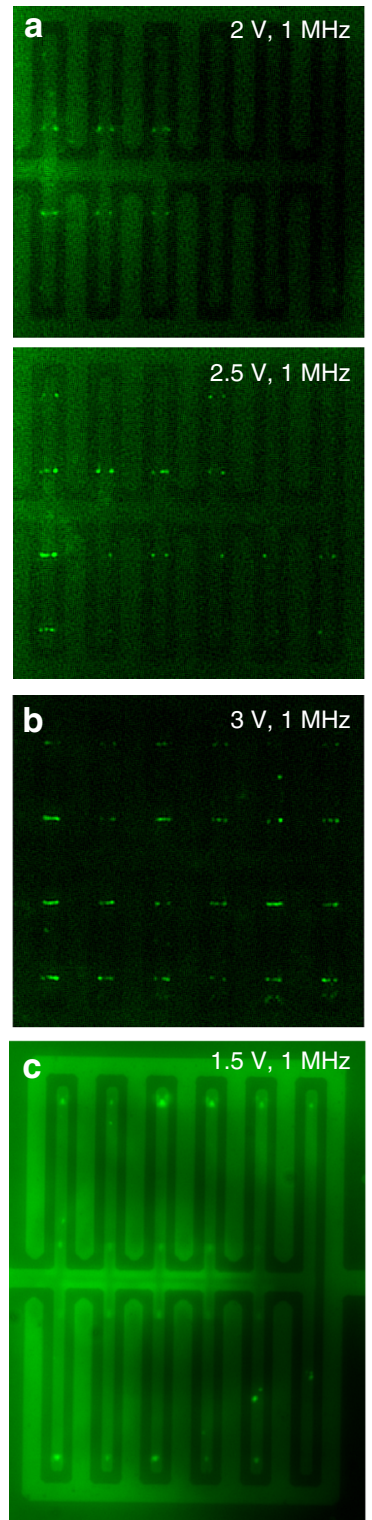
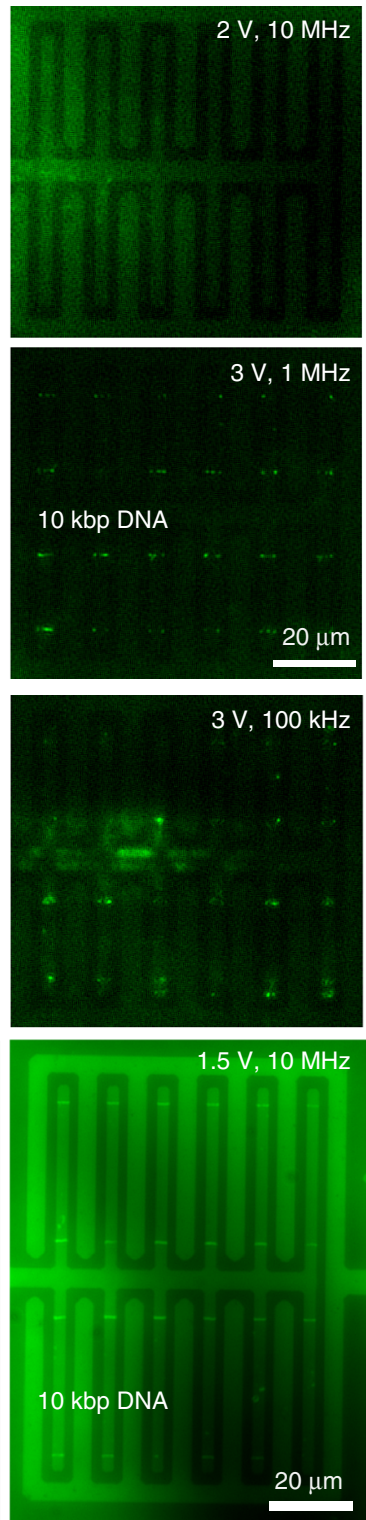
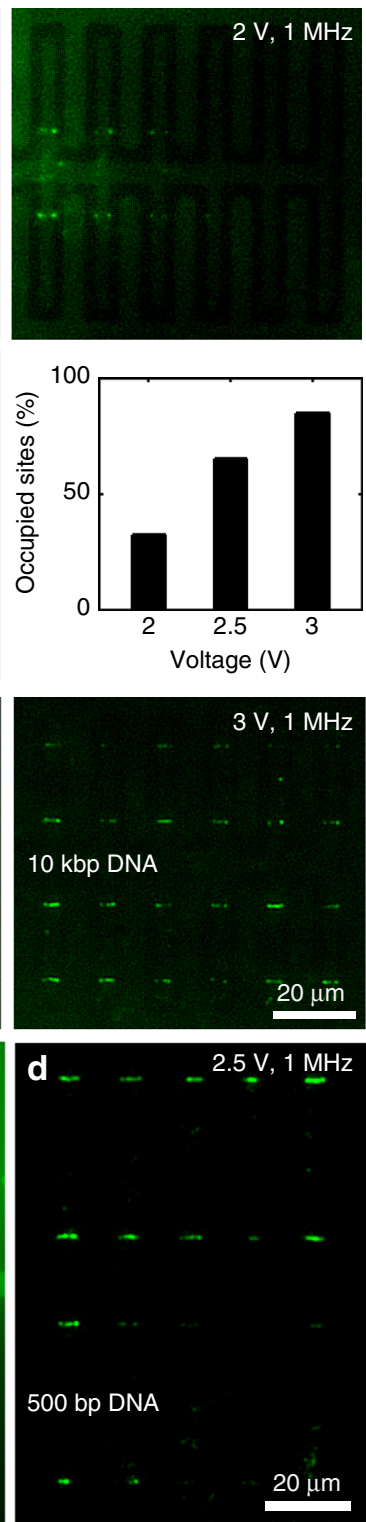

Fig. 5 DEP manipulation of DNA molecules. a Trapping and releasing of $10 \mathrm{kbp}$ DNA was observed at $1 \mathrm{MHz}$ and $10 \mathrm{MHz}$, respectively. Increasing the voltage amplitude from $2 \mathrm{~V}$ to $2.5 \mathrm{~V}$ to $3 \mathrm{~V}$, traps more DNA molecules at the trapping sites. The occupancy (\%) of the trapping sites is plotted as a function of the applied voltage. b At a lower frequency of $100 \mathrm{kHz}$, DNA (10 pM DNA in $10 \mu \mathrm{M} \mathrm{KCl}, 12 \mu \mathrm{S} / \mathrm{cm}$ ) localization near the graphene electrode could be observed instead of tight trapping along the edge, which can be reversed simply by switching to higher frequency. c At a higher solution conductivity ( $1 \mathrm{nM}$ DNA in $1 \mathrm{mM} \mathrm{KCl}, 0.93 \mathrm{mS} / \mathrm{cm}$ ), DNA localization vs the tight trapping phenomenon is observed at a higher frequency range. d 500 bp DNA molecules (threshold $\nabla|\mathbf{E}|^{2}$ an order of magnitude higher than that of the $10 \mathrm{kbp}$ DNA molecules) were trapped along the graphene edge at $2.5 \mathrm{~V}$ and $1 \mathrm{MHz}$ frequency

nature of graphene DEP manipulation (Fig. 3g). This time all 40 trapping sites were occupied as most of the released particles from the previous step stayed near the graphene edge-reducing the time of diffusion (Supplementary Movie 1). It should also be noted that in some finger electrodes more beads could be observed along the graphene edge (the red line in Fig. 3b), which could be due to (1) the entire boundary of the graphene electrode could potentially present a fringe electric field that is responsible for DEP and (2) random sharp protrusions along the edge that can enhance the electric field gradient and possibly act as a DEP trap.

Low-power DEP trapping of nanobeads with graphene electrodes. Next, we performed a voltage dependence study to determine the minimum trapping voltage $\left(V_{\min }\right)$ for $190 \mathrm{~nm}$ polystyrene beads. This is the voltage at which the DEP force is enough to overcome the Brownian motion of the nanoparticles due to their thermal energy. The amplitude of the applied voltage was increased from 400 to $700 \mathrm{mV}$ at a step interval of $50 \mathrm{mV}$. At each voltage, fluorescence images were collected for $50 \mathrm{~s}$. More beads were trapped at the trapping sites as the voltage amplitude was increased (Fig. $4 a-d$ ). To quantify this further, we measured the percentage of occupied trapping sites as a function of the applied voltage (Fig. 4e). The first data point represents $400 \mathrm{mV}$, where no trapping was observed (Fig. 4a). At $500 \mathrm{mV}$, clear trapping of polystyrene beads could be observed at multiple trapping sites (circled in Fig. 4b). At higher voltages above 700 $\mathrm{mV}$ all trapping sites were occupied. From this study, the $V_{\min }$ for $190 \mathrm{~nm}$ beads was found to be $500 \mathrm{mV}$, where at least one trapping site was fully occupied for the entire duration. 
Trapping nanodiamond particles on a 2D grid. Besides potential applications in biosensing, graphene-based DEP can be utilized for on-chip assembly and array formation of nanoscale quantum emitters such as nanodiamond particles, semiconductor nanocrystals, or plasmonic metal nanoparticles. Rapid on-chip integration of these elements with patterned graphene can be highly desirable for applications in nanosensing ${ }^{36}$ and graphene plasmonics, but is very difficult to achieve due to their nanoscale size and aggressive Brownian motion in solutions. Here we show trapping of $40 \mathrm{~nm}$ nanodiamond (ND) particles with nitrogenvacancy $(\mathrm{NV})$ centers $\left(\lambda_{\mathrm{em}}: 637 \mathrm{~nm}\right.$, Adamas Nanotechnologies) at the trapping sites on graphene (Fig. 4f). The ND particles are carboxylate-modified to ensure facile dispersion in aqueous solution. The ND particles polarize in presence of a non-uniform electric field and are attracted toward the region of strongest electric field gradient due to positive DEP. Fluorescent images $\left(\lambda_{\text {ex }}: 540-553 \mathrm{~nm}\right)$ collected after turning on the bias (amplitude $2 \mathrm{~V}$ and frequency $100 \mathrm{kHz}$ ) show ND particles localized at the trapping sites on a two-dimensional grid defined by orthogonal edges of graphene and buried metal lines.

DEP manipulation of DNA at pM concentrations. To demonstrate the utility of the graphene electrodes in capturing biomolecules, we used $10 \mathrm{kbp}$ and $500 \mathrm{bp}$ DNA molecules tagged with YOYO-1 dye $\left(\lambda_{\mathrm{ex}}: 488 \mathrm{~nm}\right)$ at a final concentration of $10 \mathrm{pM}$ in a $10 \mu \mathrm{M} \mathrm{KCl}$ solution. To predict the polarizability of the DNA molecules, a counterion fluctuation (CIF) model is often used, which depends on the redistributions of counterions around the charged sites present on the molecule. For this model to be valid, the concentration of counterions $\left(C_{\text {ions }}\right)$ present in the solution should be much greater than the total number of charged sites present across all DNA molecules, as expressed by

$$
\frac{C_{\text {ions }}}{C_{\text {DNA }}} \gg N_{\text {ions/DNA }}
$$

where $C_{\mathrm{DNA}}$ is the concentration of DNA and $N_{\text {ions/DNA }}$ represents the number of counterions required to saturate all the charged sites in a DNA molecule. Since there are two negative charges per base pair on a DNA molecule, the values of $N_{\text {ions/DNA }}$ for $10 \mathrm{kbp}$ and $500 \mathrm{bp}$ DNA are 20,000 and 1000, respectively. As $C_{\text {ions }} / C_{\text {DNA }}\left(=10^{6}\right)$ is much higher than $N_{\text {ions/DNA }}$, the criterion in equation 7 is satisfied. The trapping force however must still be enough to overcome the thermal drag force (equation 2), which is contributed by diffusion. The DEP force on a DNA molecule can be expressed by:

$$
\vec{F}_{\mathrm{DEP}}=\frac{1}{4} \alpha \nabla|\mathbf{E}|^{2}
$$

where $\alpha$ is the real part of the polarizability of the DNA molecule $^{37}$. The polarizability values were estimated from previously reported empirical findings that correspond to $\sim 3.48 \times 10^{-31} \mathrm{Fm}^{2}$ and $\sim 1.00 \times 10^{-31} \mathrm{Fm}^{2}$ for the $10 \mathrm{kbp}$ and $500 \mathrm{bp}$ DNA, respectively $^{37}$. Next, we calculated the threshold $\nabla|\mathbf{E}|^{2}$ by equating the DEP force (equation 8 ) with the thermal force (equation 2). The radius of gyration $(\mathrm{Rg})$ of $10 \mathrm{kbp}$ DNA with persistence length 50 $\mathrm{nm}$, calculated using a worm-like chain model ${ }^{38,39}$, is $238 \mathrm{~nm}$ that corresponds to an $F_{\mathrm{T}}$ value of $14.4 \mathrm{fN}$ (using equation 2). The threshold $\nabla|\mathbf{E}|^{2}$ to trap a $10 \mathrm{kbp}$ DNA molecules is $\sim 1.66 \times 10^{17}$ $\mathrm{V}^{2} \mathrm{~m}^{-3}$ (using equation 8). For $500 \mathrm{bp}$ DNA with $R \mathrm{~g}$ of $53 \mathrm{~nm}$, $\left(F_{\mathrm{T}} \sim 64.7 \mathrm{fN}\right)$, the threshold $\nabla|\mathbf{E}|^{2}$ is $\sim 2.59 \times 10^{18} \mathrm{~V}^{2} \mathrm{~m}^{-3}$.

Figure 5 a shows trapping and releasing of DNA molecules at an applied voltage of amplitude $2 \mathrm{~V}$. A frequency of $1 \mathrm{MHz}$ was used to capture the DNA molecules at the trapping sites. The efficiency of trapping goes down at higher frequencies, as the counterions present in the solution do not have enough time to redistribute in each cycle of the $\mathrm{AC}$ bias. Thus at $10 \mathrm{MHz}$ the DNA molecules lose their polarizability and are released into the solution. The threshold $\nabla|\mathbf{E}|^{2}$ to trap a $10 \mathrm{kbp}$ DNA molecule is $1.66 \times 10^{17} \mathrm{~V}^{2} \mathrm{~m}^{-3}$, which is trivial to achieve in graphene electrodes (Fig. 2d). However, a slightly higher voltage $(2 \mathrm{~V})$ was applied to achieve DEP manipulation, as the concentration of DNA used in this experiment was fairly low (10 pM). By applying higher voltages, it is possible to trap more number of DNA molecules. This was further demonstrated by applying higher voltage amplitudes of $2.5 \mathrm{~V}$ and $3 \mathrm{~V}$ that improved the DNA capture efficiency as evident from the increased number of occupied trapping sites. We also measured the occupancy of trapping sites as a function of the applied voltage (Fig. 5a). At $2 \mathrm{~V}$, around $32.5 \%$ of the trapping sites were occupied, which was increased to $65 \%$ at $2.5 \mathrm{~V}$ and $85 \%$ at $3 \mathrm{~V}$.

So far, we showed DEP manipulation using high frequency AC fields $(1-10 \mathrm{MHz})$. Reducing the frequency to $100 \mathrm{kHz}$, results in an interesting localization of the DNA molecules near the graphene edge but the molecules are not tightly trapped at the edge (Fig. 5b). This phenomenon could be explained by the generation of a fluid flow at lower frequencies due to the formation of electrical double layers near the electrode surface. However, this mechanism depends on the charge relaxation frequency $(f)$ of the system, which is given by

$$
f=\frac{\sigma_{\mathrm{m}}}{2 \pi \varepsilon_{\mathrm{m}}}
$$

For a solution of conductivity $12 \mu \mathrm{S} / \mathrm{cm}(10 \mu \mathrm{M} \mathrm{KCl}$ solution, measured by B-771 LAQUAtwin, Horiba Scientific), the charge relaxation frequency is $270 \mathrm{kHz}$ (from equation 9). Hence, while operating at $100 \mathrm{kHz}$, the system has enough time to create electrical double layers, which can generate fluid flow due to electroosmosis. This effect can also be switched to the case of tight trapping along the edge of graphene, simply by switching to higher frequencies. Figure $5 \mathrm{~b}$ shows the switching between these two effects in a reversible fashion with tight trapping at $1 \mathrm{MHz}$ and localization near graphene edge at $100 \mathrm{kHz}$ (Supplementary Movie 2). Increasing the solution conductivity will also increase the charge relaxation frequency. For instance, using a solution of higher conductivity with $1 \mathrm{nM}$ DNA in $1 \mathrm{mM} \mathrm{KCl}$ (conductivity $0.93 \mathrm{mS} / \mathrm{cm}$ ), increases the relaxation frequency to $21 \mathrm{MHz}$. In this case, we can observe a similar DNA localization effect near the graphene edge even at $1 \mathrm{MHz}$ (Fig. 5c). Switching to higher frequency traps the DNA molecules in a tight fashion along the graphene edge. The increased brightness of the overall background as well as the reduced operating voltage $(1.5 \mathrm{~V})$ is attributed to the $100 \times$ higher concentration of the DNA molecules.

$500 \mathrm{bp}$ DNA was used to demonstrate trapping of smaller DNA strands (Fig. 5d). The threshold $\nabla|\mathbf{E}|^{2}$ to capture a $500 \mathrm{bp}$ DNA molecule is $2.59 \times 10^{18} \mathrm{~V}^{2} \mathrm{~m}^{-3}$, which is an order of magnitude higher than the threshold $\nabla|\mathbf{E}|^{2}$ required to trap 10 kbp DNA molecules. From Fig. $2 \mathrm{~d}$ we estimate that theoretically a $500 \mathrm{bp}$ DNA molecule can be captured at a distance of $250 \mathrm{~nm}$ away from the graphene edge while applying an AC bias of $1.4 \mathrm{~V}$ amplitude. However, here we chose to work with a bias of amplitude $2.5 \mathrm{~V}$ to increase the range of trapping. Also, as $500 \mathrm{bp}$ DNA molecules have an $R g$ of $53 \mathrm{~nm}$, it was hard to observe single DNA molecule trapping at the trapping sites. Hence, we waited until trapping was observed along the entire edge of graphene electrode, which could be achieved within one minute.

Stability of biomolecules is dependent on the temperature of the surrounding environment. We investigated the expected temperature rise in our system based on Joule heating from the 
relation $\Delta T_{\mathrm{S}} \sim \sigma_{\mathrm{m}} V^{2} / 2 k$ (where $\Delta T_{\mathrm{S}}$ is the rise in solution temperature and $k$ is the thermal conductivity). As we used low voltages and low-conductivity solutions in our system, the expected temperature rise is minimal. Even for the case where we expect highest $\Delta T_{\mathrm{S}}$ (while using $1 \mathrm{mM} \mathrm{KCl}$ solution with conductivity $0.93 \mathrm{mS} / \mathrm{cm}$ ), the temperature rise within the system should be less than $1{ }^{\circ} \mathrm{C}$. For the case where we used highest operating voltage (amplitude $3 \mathrm{~V}$ ), the expected temperature rise is $<0.05^{\circ} \mathrm{C}$.

\section{Discussion}

Monolayer graphene can generate ultra-strong dielectrophoresis forces and outperform conventional metal electrodes that are orders of magnitude thicker. We have presented the concept of graphene-edge DEP and constructed a practical device platform to realize it. In our scheme, the critical dimension of graphene electrodes for DEP is controlled by its natural thickness and the insulator deposited by ALD, thus our approach is scalable and highly reproducible at the wafer scale. Using arrays of electrodes, we can selectively trap and position nanoparticles and DNA molecules precisely along the atomically sharp edges of graphene. This could facilitate performing of biological assays at low concentrations as well as exploring molecular interactions and conformational changes in confined space. We also demonstrated trapping of DNA molecules from 10 pM solution and on-chip assembly and precise positioning of nanodiamonds. Notably all of these rapid DEP manipulation steps were performed using monolayer graphene around or below $1 \mathrm{~V}$, which makes the lowpower graphene-edge DEP attractive for many applications. For example, edge trapping of biomolecules can be readily integrated with graphene nanoresonators $11,40,41$ or tapered nanotips 7,8 wherein field hotspots are located along the edges, enabling tunable mid-IR spectroscopy of ultralow-concentration molecules using graphene plasmons. Our wafer-scale chip platform can also be used for nano-positioning quantum emitters to build nanophotonic circuits or single-photon source arrays ${ }^{42}$. While we used straight edges of graphene in this work, DEP can also be performed with conductive nanopores ${ }^{43,44}$, presenting new opportunities for single DNA translocation and analysis ${ }^{45-48}$. Beyond graphene, atomically sharp edges of other $2 \mathrm{D}$ materials ${ }^{49}$ can also be utilized for ultra-strong DEP, providing a practical route to building tunable biosensors.

\section{Methods \\ Device fabrication. Our graphene-edge DEP chip was fabricated starting from a thick layer of $\mathrm{SiO}_{2}(300 \mathrm{~nm})$ thermally grown on $\mathrm{Si}$ substrate. The gate electrode patterns were exposed and developed using photolithography, followed by a combination of reactive ion etching and wet etching on the $\mathrm{SiO}_{2}$ layer. A metal- lization layer of $\mathrm{Ti} / \mathrm{Pd}(10 / 40 \mathrm{~nm})$ was then deposited by electron-beam evapora- tion and lifted off. Atomic layer deposition of $\mathrm{HfO}_{2}$ at $300{ }^{\circ} \mathrm{C}$ was applied to form a dielectric layer $(8 \mathrm{~nm})$, which was annealed in nitrogen at $400{ }^{\circ} \mathrm{C}$ for 5 minutes. A via layer was patterned using photolithography, and then the $\mathrm{HfO}_{2}$ in localized regions above the $\mathrm{Pd}$ metal was reactive-ion etched in $\mathrm{SF}_{6}$ for $30-60 \mathrm{~s}$. The via window through the dielectric layer is intended to provide the ability to subse- quently contact the gate electrode with additional contact metallization. Single- layer graphene grown by chemical vapor deposition (CVD) was transferred onto the dielectric layer using a wet transfer process, patterned by photolithography, and then etched using an $\mathrm{O}_{2}$ plasma. Ohmic contacts were patterned next using photolithography, and $\mathrm{Cr} / \mathrm{Au}(10 / 80 \mathrm{~nm})$ was deposited using electron-beam evaporation and lifted off. Finally, for the convenience of the device measurement, a thick metal layer of $\mathrm{Cr} / \mathrm{Al}(10 / 300 \mathrm{~nm})$ was patterned and deposited to form low- resistance electrical leads from the gate and Ohmic contacts to the contact pads for electrical probing.}

DEP experiments. A solution volume of $\sim 10 \mu \mathrm{l}$ was placed on top of graphene electrodes, confined within a reservoir made in adhesive tape. A cover slip was placed to avoid any unwanted evaporation. A probe station was used to apply an AC bias across the contact pads by a function generator (HP $33120 \mathrm{~A}$ ). The peak amplitude of the voltage used was in the range of 1-3 V. Depending on the particle, the frequency of the AC bias was optimized for polystyrene, DNA molecules, and nanodiamonds. For the polystyrene and ND particles, water was used as a surrounding medium (conductivity $\sim 4 \mu \mathrm{S} / \mathrm{cm}$ ), whereas $10 \mu \mathrm{M} \mathrm{KCl}$ solution (conductivity $\sim 12 \mu \mathrm{S} / \mathrm{cm}$ ) was used for DNA.

Fluorescence microscopy. Fluorescent nanoparticles used in this work are $190 \mathrm{~nm}$ diameter carboxylate-modified polystyrene beads $\left(\lambda_{\mathrm{ex}}: 470 \mathrm{~nm}, \lambda_{\mathrm{em}}: 525 \mathrm{~nm}\right.$, Bangs Laboratories); high-pressure, high-temperature monocrystalline and carboxylatemodified nanodiamonds of type $\mathrm{lb}$ containing 10-15 NV centers, with average volumetric size of $40 \mathrm{~nm}$ ( $\lambda_{\mathrm{em}}: 637 \mathrm{~nm}$, Adamas Nanotechnologies); and YOYO-1 labeled DNA molecules of length $10 \mathrm{kbp}$ and $500 \mathrm{bp}\left(\lambda_{\mathrm{ex}}: 491 \mathrm{~nm}\right)$. A laser driven light source (Energetiq) was illuminated through appropriate filters to excite fluorescence and the emitted light from the solution was collected through another filter. A $50 \times$ objective was used to observe nanoparticles and DNA molecules while using an image collection time of $200 \mathrm{~ms}$ for $190 \mathrm{~nm}$ beads, $1 \mathrm{~s}$ for the nanodiamond particles, $500 \mathrm{~ms}$ for the $10 \mathrm{kbp}$ DNA and $1 \mathrm{~s}$ for the $500 \mathrm{bp}$ DNA molecules. Fluorescent images were collected at regular intervals using a Photometrics CoolSNAP HQ2 CCD camera and Micro-Manager software, and they were further analyzed using ImageJ software.

Data availability. The data that supports the finding of this study are available from the corresponding author upon request.

Received: 16 June 2017 Accepted: 5 October 2017

Published online: 30 November 2017

\section{References}

1. Geim, A. K. \& Novoselov, K. S. The rise of graphene. Nat. Mater. 6, 183-191 (2007).

2. Neto, A. H. C., Guinea, F., Peres, N. M. R., Novoselov, K. S. \& Geim, A. K. The electronic properties of graphene. Rev. Mod. Phys. 81, 109-162 (2009).

3. Bonaccorso, F., Sun, Z., Hasan, T. \& Ferrari, A. C. Graphene photonics and optoelectronics. Nat. Photon. 4, 611-622 (2010).

4. Koester, S. J. High quality factor graphene varactors for wireless sensing applications. Appl. Phys. Lett. 99, 163105 (2011).

5. Deen, D. A., Olson, E. J., Ebrish, M. A. \& Koester, S. J. Graphene-Based Quantum Capacitance Wireless Vapor Sensors. IEEE Sensors J. 14, 1459-1466 (2014).

6. Grigorenko, A. N., Polini, M. \& Novoselov, K. S. Graphene plasmonics. Nat. Photon. 6, 749-758 (2012).

7. Chen, J. et al. Optical nano-imaging of gate-tunable graphene plasmons. Nature 487, 77-81 (2012).

8. Fei, Z. et al. Gate-tuning of graphene plasmons revealed by infrared nanoimaging. Nature 487, 82-85 (2012).

9. Low, T. \& Avouris, P. Graphene plasmonics for terahertz to mid-infrared applications. ACS Nano 8, 1086-1101 (2014).

10. Low, T. et al. Polaritons in layered two-dimensional materials. Nat. Mater. 16, 182-194 (2017).

11. Rodrigo, D. et al. Mid-infrared plasmonic biosensing with graphene. Science 349, 165-168 (2015).

12. Li, W., Chung, J. K., Lee, Y. K. \& Groves, J. T. Graphene-templated supported lipid bilayer nanochannels. Nano Lett. 16, 5022-5026 (2016).

13. $\mathrm{Xu}, \mathrm{S}$. et al. Real-time reliable determination of binding kinetics of DNA hybridization using a multi-channel graphene biosensor. Nat. Commun. 8, 14902 (2017).

14. Novotny, L. \& van Hulst, N. F. Antennas for light. Nat. Photon. 5, 83-90 (2011).

15. Beams, R., Bharadwaj, P. \& Novotny, L. Electroluminescence from graphene excited by electron tunneling. Nanotechnology 25, 055206 (2014).

16. Juan, M. L., Gordon, R., Pang, Y., Eftekhari, F. \& Quidant, R. Self-induced backaction optical trapping of dielectric nanoparticles. Nat. Phys. 5, 915-919 (2009).

17. Juan, M. L., Righini, M. \& Quidant, R. Plasmon nano-optical tweezers. Nat. Photon. 5, 349-356 (2011).

18. Maragò, O. M., Jones, P. H., Gucciardi Pietro, G., Volpe, G. \& Ferrari, A. C. Optical trapping and manipulation of nanostructures. Nat. Nanotechnol. 8, 807-819 (2013)

19. Wu, M. C. Optoelectronic tweezers. Nat. Photon. 5, 322-324 (2011).

20. Zhang, S., Juvert, J., Cooper, J. M. \& Neale, S. L. Manipulating and assembling metallic beads with Optoelectronic Tweezers. Sci. Rep. 6, 32840 (2016).

21. Eftekhari, F. et al. Nanoholes as nanochannels: flow-through plasmonic sensing. Anal. Chem. 81, 4308-4311 (2009).

22. Yanik, A. A., Huang, M., Artar, A., Chang, T.-Y. \& Altug, H. Integrated nanoplasmonic-nanofluidic biosensors with targeted delivery of analytes. Appl. Phys. Lett. 96, 021101 (2010). 
23. Cho, H., Lee, B., Liu, G. L., Agarwal, A. \& Lee, L. P. Label-free and highly sensitive biomolecular detection using SERS and electrokinetic preconcentration. Lab. Chip. 9, 3360-3363 (2009).

24. Pohl, H. A. Dielectrophoresis: the behavior of neutral matter in nonuniform electric fields (Cambridge University Press, Cambridge, UK, 1978).

25. Ndukaife, J. C. et al. Long-range and rapid transport of individual nano-objects by a hybrid electrothermoplasmonic nanotweezer. Nat. Nanotechnol. 11, 53-59 (2016).

26. Morgan, H. \& Green, N. G. AC Electrokinetics: Colloids and Nanoparticles (Research Studies Press, Philadelphia, USA, 2003).

27. Jose, J. et al. Individual template-stripped conductive gold pyramids for tipenhanced dielectrophoresis. ACS Photon. 1, 464-470 (2014).

28. Barik, A., Chen, X. \& Oh, S.-H. Ultralow-power electronic trapping of nanoparticles with sub-10 nm gold nanogap electrodes. Nano Lett. 16, 6317-6324 (2016).

29. Kuzyk, A. Dielectrophoresis at the nanoscale. Electrophoresis 32, 2307-2313 (2011).

30. Zheng, L., Li, S., Brody, J. P. \& Burke, P. J. Manipulating nanoparticles in solution with electrically contacted nanotubes using dielectrophoresis. Langmuir 20, 8612-8619 (2004)

31. Tuukkanen et al. Carbon nanotubes as electrodes for dielectrophoresis of DNA. Nano Lett. 6, 1339-1343 (2006).

32. Xie, S., Jiao, N., Tung, S. \& Liu, L. Fabrication of SWCNT-graphene field-effect transistors. Micromachines 6, 1317-1330 (2015).

33. Squires, T. M., Messinger, R. J. \& Manalis, S. R. Making it stick: convection, reaction and diffusion in surface-based biosensors. Nat. Biotechnol. 26, 417-426 (2008).

34. Lee, B., Moon, T., Kim, T.-G., Choi, D.-K. \& Park, B. Dielectric relaxation of atomic-layer-deposited $\mathrm{HfO}_{2}$ thin films from $1 \mathrm{kHz}$ to $5 \mathrm{GHz}$. Appl. Phys. Lett. 87, 012901 (2005)

35. Zhao, C., Zhao, C. Z., Werner, M., Taylor, S. \& Chalker, P. Dielectric relaxation of high-k oxides. Nanoscale Res. Lett. 8, 456 (2013).

36. Geiselmann, M. et al. Three-dimensional optical manipulation of a single electron spin. Nat. Nanotechnol. 8, 175-179 (2013).

37. Tuukkanen, S. et al. Trapping of $27 \mathrm{bp}-8 \mathrm{kbp}$ DNA and immobilization of thiol-modified DNA using dielectrophoresis. Nanotechnology 18, 295204 (2007).

38. Mantelli, S., Muller, P., Harlepp, S. \& Maaloum, M. Conformational analysis and estimation of the persistence length of DNA using atomic force microscopy in solution. Soft Matter 7, 3412-3416 (2011).

39. Wenner, J. R., Williams, M. C., Rouzina, I. \& Bloomfield, V. A. Salt Dependence of the Elasticity and Overstretching Transition of Single DNA Molecules. Biophys. J. 82, 3160-3169 (2002).

40. Yan, H. et al. Damping pathways of mid-infrared plasmons in graphene nanostructures. Nat. Photon. 7, 394-399 (2013).

41. Nikitin, A. Y. et al. Real-space mapping of tailored sheet and edge plasmons in graphene nanoresonators. Nat. Photon. 10, 239-243 (2016).

42. Pelton, M. Modified spontaneous emission in nanophotonic structures. Nat. Photon. 9, 427-435 (2015)

43. Barik, A. et al. Dielectrophoresis-enhanced plasmonic sensing with gold nanohole arrays. Nano Lett. 14, 2006-2012 (2014).

44. Freedman, K. J. et al. Nanopore sensing at ultra-low concentrations using single-molecule dielectrophoretic trapping. Nat. Commun. 7, 10217 (2016).

45. Merchant, C. A. et al. DNA translocation through graphene nanopores. Nano Lett. 10, 2915-2921 (2010).

46. Schneider, G. F. et al. Tailoring the hydrophobicity of graphene for its use as nanopores for DNA translocation. Nat. Commun. 4, 2619 (2013).
47. Traversi, F. et al. Detecting the translocation of DNA through a nanopore using graphene nanoribbons. Nat. Nanotechnol. 8, 939-945 (2013).

48. Crick, C. R., Sze, J. Y. Y., Rosillo-Lopez, M., Salzmann, C. G. \& Edel, J. B. Selectively Sized Graphene-Based Nanopores for in Situ Single Molecule Sensing. ACS Appl. Mater. Interfaces 7, 18188-18194 (2015).

49. Feng, J. et al. Identification of single nucleotides in MoS2 nanopores. Nat. Nanotechnol. 10, 1070-1076 (2015).

\section{Acknowledgements}

This work was supported by the National Science Foundation (NSF ECCS No. 1610333 to S.-H.O.); The Minnesota Partnership for Biotechnology and Medical Genomics (A.B., S.-H.O., Y.Z., and S.J.K.); J.B.E. acknowledges support from the EPSRC and ERC (Starter and Consolidator grants). A.B. acknowledges support from the University of Minnesota Doctoral Dissertation Fellowship. Device fabrication was performed at the University of Minnesota Nanofabrication Center, which receives support from the NSF through the National Nanotechnology Coordinated Infrastructure (NNCI) program, and the Characterization Facility, which has received capital equipment funding from the NSF through the MRSEC program under award no. DMR-1420013. Computational modeling was carried out using software provided by the University of Minnesota Supercomputing Institute. The authors thank Jonah Shaver for helping with the optics setup and Seon Namgung for helping with sample preparation.

\section{Author contributions}

A.B. performed DEP trapping experiments. Y.Z. and S.J.K. performed chip design and fabrication. R.G. and T.L. performed theoretical calculations. B.P.N. and J.B.E. prepared DNA samples. S.-H.O. conceived and supervised the research. All the authors contributed to the analysis of the data and writing of the manuscript.

\section{Additional information}

Supplementary Information accompanies this paper at doi:10.1038/s41467-017-01635-9.

Competing interests: The authors declare no competing financial interests.

Reprints and permission information is available online at http://npg.nature.com/ reprintsandpermissions/

Publisher's note: Springer Nature remains neutral with regard to jurisdictional claims in published maps and institutional affiliations.

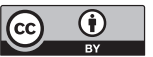

Open Access This article is licensed under a Creative Commons Attribution 4.0 International License, which permits use, sharing, adaptation, distribution and reproduction in any medium or format, as long as you give appropriate credit to the original author(s) and the source, provide a link to the Creative Commons license, and indicate if changes were made. The images or other third party material in this article are included in the article's Creative Commons license, unless indicated otherwise in a credit line to the material. If material is not included in the article's Creative Commons license and your intended use is not permitted by statutory regulation or exceeds the permitted use, you will need to obtain permission directly from the copyright holder. To view a copy of this license, visit http://creativecommons.org/ licenses/by/4.0/.

(c) The Author(s) 2017 